\title{
Methyl Torsion: An Overview
}

\section{Srivastava AK* and Swasti Saxena}

Applied Physics Department, Sardar Vallabhbhai National Institute of Technology, India

*Corresponding author: Ankit K Srivastava, Applied Physics Department, Sardar

Vallabhbhai National Institute of Technology, Surat, Gujarat, India, 395007, Tel: +91-

\section{Editorial}

Volume 3 Issue 2

Received Date: July 11, 2019

Published Date: July 22, 2019

DOI: $10.23880 /$ macij-16000141

9769336195; Email: pushpankit@gmail.com

\section{Introduction}

In methylated molecules potential having multi minima arises due to the methyl group internal rotation about a single bond which encounters some stable as well as unstable conformations. In past years, the hindered motion of methyl group appeared as a quantum mechanical problem. Experimental as well as theoretical investigations have been carried out to understand the nature of the barrier due to the torsional motion using accurate prediction from ab initio calculations and FTIR, microwave and RAMAN spectroscopy. The barrier height for toluene is found to be of the order of a few wavenumbers whereas for ethane it is of the order of thousand wavenumbers. It is highly sensitive towards the chemical as well as electronic environment adjacent to the methyl group. Therefore, the methyl group internal rotation is an important subject to study the conformation and inter or intra-molecular dynamics and hence has attracted the attention from both physicists and chemists.

Due to the low frequency vibration of torsional motion, the issues for observing these vibration experimentally is a challenging task. Torsional bands are often very close to the origin and thus lie within the origin band at room temperature due to large bandwidth. Thus it is important to adopt spectroscopic technique that provides relatively high resolution spectrum. Supersonic jet spectroscopic technique is the most promising technique as the low frequency vibrations near the origin band of the excitation spectrum of the molecular system in gaseous form can be resolved using this technique. The resolution of the obtained excitation spectrum of the molecular system is very high because the spectrum is obtained for cold isolated molecule and is free from the Doppler broadening. The barrier to internal rotation about a single bond is an important tool to monitor the conformation of the selected molecules and also determines the structural as well as functional characteristics of carbohydrates, nucleic acids, proteins.
Methyl groups are deeply involved in pharmacological field [1] as their presence can easily change not only the effectiveness and nature but also the inhibition property of molecule [2]. The effect of the presence of methyl group can be easily understood by studying DNA and RNA. Though presence of a methyl group on pyrimidine ring signifies the only structural difference in DNA and RNA (thymine and uracil), but there are many other differences between these two nucleic acids which exhibit due to the presence of methyl group in the structure of DNA. The presence of methyl group leads to some biological processes such as replication, transcription, molecular recognition, and cell metabolism [3-5]. The methyl group can be used in metabolism through oxidation in alcohol and aldehyde. It can also be used for the improvement of metabolic stability of some molecules which are used in the medical field. These investigations can help to develop the understanding of the behavior of methyl group.

The internal rotation of methyl group in nitrogenous heterocyclic molecules has its importance as these molecules are one of the building blocks in many biomolecules. To understand the photophysical and photochemical properties of these molecules in isolation system like proteins, the study of methyl internal rotation is focussed as it controls the rate of intramolecular vibrational (IVR) energy redistribution. In this regard, some work has been done on different nitrogenous heterocyclic molecules using supersonic jet spectroscopy previous years [6-10]. It was found that the skeletal relaxation plays an important role in determining the origin of the barrier and also the asymmetric substitution of methyl group as in 4-methylstyrene [10], can be used to tailor the $\pi$ electron conjugation between the ring and vinyl group which can further be used to control the barrier. Hence it has important implications in chemical and biological processes. From the spectroscopic study of these molecules, the change in torsional behaviour of 


\section{Medicinal \& Analytical Chemistry International Journal}

different substitutions as well as the change in periodicity of methyl torsional potential can be determined.

Though the barrier height, shape and the minimum energy conformation of the methyl group depends on the electronic distributions and the interactions within the molecular system, the nature of forces for origin of barrier in various molecules is still a matter of intense research for biomolecules. Various properties have been proposed to explain the behaviour of methyl torsion in different molecules, but a generalized model is still lacking which can combine the diverse behaviour of the methyl conformation and its internal rotation. Thus, it needs detailed study to understand the methyl torsion and the barrier formation due to it.

\section{References}

1. Bazzini P, Wermuth CG (2008) The Practice of Medicinal Chemistry $3^{\text {rd }}($ Edn). Academic Press, New York.

2. Hoffman WF, Alberts AW, Anderson PS, Chen JS, Smith RL, et al. (1986) 3-hydroxy-3- methylglutarylcoenzyme a reductase inhibitors. 4. Side chain ester derivatives of mevinolin. J Medi Chem 29(5): 849852.

3. Umezawa Y, Nishio M, (2002) Thymine-methyl/ $\pi$ interaction implicated in the sequence-dependent deformability of DNA. Nucleic Acids Res 30(10): 2183-2192.
4. Singleton CK, Martin PR (2001) Molecular mechanisms of thiamine utilization. Curr Mole Medi 1(2): 197-207.

5. Massey V (2000) The chemical and biological versatility of riboflavin. Biochem Soc Transac 28(4): 283-296.

6. Sinha RK, Pradhan B, Wategaonkar S, Singh BP, Kundu T (2007) Spectroscopic studies on methyl torsional behaviour in 1-methyl-2(1h)-pyridone, 1methyl-2(1h)-pyridinimine, and 3-methyl-2(1h)pyridone. I. excited state. J Chem Phys 126(11): 114312.

7. Pradhan B, Sinha RK, Singh BP, Kundu T (2007) Origin of methyl torsional barrier in 1-methyl-2(1h)pyridinimine and 3-methyl-2(1h)-pyridone: II. ground state. J Chem Phys 126(11): 114313.

8. Sinha RK, Singh BP, Kundu T (2008) Origin of threefold methyl torsional potential in methylindoles. Theo Chem Account 121(1): 59-70.

9. Pradhan B, Singh BP, Nandi CK, Chakraborty T, Kundu $T$ (2005) Origin of methyl torsional barrier in 1methyl-2-(1h)-pyridone. J Chem Phys 122(20): 204323.

10. Sinha RK, Pradhan B, Singh BP, Kundu T, Biswas $P$, et al. (2006) Origin of threefold symmetric torsional potential of methyl group in 4-methylstyrene. J Chem Phys 124(14): 144316. 